\title{
Dirac Equation at Finite Temperature
}

\author{
Xiang-Yao $\mathrm{Wu}^{a}{ }^{1}$, Bo-Jun Zhang ${ }^{a}$, Xiao-Jing $\mathrm{Liu}^{a}$, Nuo $\mathrm{Ba}^{a}$ \\ Yi-Heng $\mathrm{Wu}^{a}$, Si-Qi Zhang ${ }^{a}$, Jing $\mathrm{Wang}^{a}$ and Chun-Hong $\mathrm{Li}^{a}$ \\ a. Institute of Physics, Jilin Normal University, Siping 136000, China
}

\begin{abstract}
In this paper, we propose finite temperature Dirac equation, which can describe the quantum systems in an arbitrary temperature for a relativistic particle of spin- $1 / 2$. When the temperature $T=0$, it become Dirac equation. With the equation, we can study the relativistic quantum systems in an arbitrary temperature.
\end{abstract}

PACS numbers: 03.65.-w; 11.10.Wx

Keywords: Dirac equation; Finite temperature

\footnotetext{
${ }^{1}$ E-mail:wuxy2066@163.com
} 


\section{Introduction}

We know that the studies of physical systems at finite temperature have led to many interesting properties such as phase transitions, black body radiation etc. However, the study of complicated quantum mechanical systems at finite temperature has had a systematic development only in the past few decades. There are now well developed and well understood formalisms to describe finite temperature field theories, as they are called. In fact, as we know now, there are three distinct, but equivalent formalisms [1-3] to describe such theories and each has its advantages and disadvantages. But, the important point to note is that we now have a systematic method of calculating thermal averages in quantum field theory. Quantum field theory at finite temperature is the relativistic generalization of finite temperature non-relativistic quantum statistical mechanics [4]. There it is known that quantum effects are only important at low temperature. More precisely the important parameter is the ratio of the thermal wave-length and the length scale which characterizes the variation of the potential. Only when this ratio is large are quantum effects important. Increasing the temperature is at leading order equivalent to decrease $\hbar$. Note that, from the point of view of the path integral representation of quantum mechanics, the transition from quantum to classical statistical mechanics appears as a kind of dimensional reduction: in the classical limit path integrals reduce to ordinary integrals.

With the finite temperature quantum field theories, we can now study questions such as phase transitions involving symmetry restoration in theories with spontaneously broken symmetry [5]. We can study the evolution of the universe at early times which clearly is a system at high temperature. More recently, even questions such as the chiral symmetry breaking phase transition [6] have drawn a lot of attention in view of the planned experiments involving heavy ion collisions. This would help us understand properties of the quark-gluon plasma better.

Quantum field theories at finite temperature are very challenging also from the more theoretical point of view. There is a real-time as well as an imaginary-time formalism, the first describing dynamical and the second equilibrium properties [7]. Many fundamental issues and problems are unsolved so far or require a deeper understanding. Quantum field theories are subject to enhanced complexities compared to zero temperature and 
zero density. In this paper, we define the covariation microscopic entropy for a relativistic particle of spin- $1 / 2$, and then obtain a new finite temperature Dirac equation, which can study the relativistic quantum systems in an arbitrary temperature, and can further develop to become finite temperature quantum field theory.

\section{The concept of microscopic and macroscopic en- tropy}

In thermodynamics, the infinitesimal entropy change $d S$ of a system is defined by

$$
d S=\frac{\delta Q}{T},
$$

where $\delta Q$ is a transfer of heat between the composite system and an external reservoir at the temperature $T$. In a finite change of state from $i$ to $j$, the entropy change is $S_{j}-S_{i}$,

$$
S_{j}-S_{i}=\int_{i}^{j} \frac{\delta Q}{T}
$$

The entropy of a system is a function of the thermodynamic coordinates whose change

is equal to the integral of $\frac{\delta Q}{T}$ between the terminal states, integrated along any reversible path connecting the two states.

By integrating (1) around a reversible cycle, so that the initial and final entropies are the same. For a reversible cycle, we get

$$
\oint \frac{\delta Q}{T}=0 .
$$

In quantum statistics, the entropy $S$ is defined by

$$
S=-k_{B} \operatorname{Tr}(\rho \ln \rho)
$$

where $\rho=|\psi\rangle\langle\psi|$ is the density matrix, $k_{B}$ is the Boltzmann constant.

In classical statistics, the entropy is defined by the Boltzmann

$$
S=k_{B} \ln W=-k_{B} \ln \rho,
$$

where $W$ is the total number of the possible microscopic states, and $\rho=\frac{1}{W}$ is probability of every state. 
From (5), we know that the macroscopic entropy is from the state distribution of a large number of particles. According to the viewpoint of macroscopic entropy, as the single particle hasn't state distribution, the single particle hasn't entropy.

In the viewpoint of quantum mechanics, a microscopic particle has wave-particle duality, and the wave nature is described by wave function. Obviously, the wave functions have the nature of distribution. So, a single particle has microscopic entropy, and we define its microscopic entropy $S$ as

$$
S=-k_{w} \ln |\psi(\vec{r}, t)|^{2}
$$

Where $k_{w}$ is a constant, which will be confirmed by experiment.

For a relativistic particle of spin-1/2, we should consider the covariation. In Eq. (6), we should replace the probability density $|\psi|^{2}$ with Lorentz scalar $\bar{\psi} \psi$, and can obtain the microscopic entropy $S$ for a relativistic particle of spin-1/2, they are

$$
S=-k_{w} \ln (\bar{\psi} \psi)
$$

\section{The relation between a particle energy and tem- perature}

Based on the first law of thermodynamics, there is

$$
d U=d W+d Q
$$

where $d U$ is the infinitesimal change of system internal energy, $d W$ is the infinitesimal work doing by surrounding, and $d Q$ is the infinitesimal heat absorbed from surrounding. In this paper, we should study the quantum thermodynamics property of microscopic particles, i.e., finite temperature quantum theory of $\mathrm{N}$-particle. When $d W=0$, the (9) becomes

$$
d U-d Q=0
$$

For the point particle system of $\mathrm{N}$-particle, the internal energy $U$ is

$$
U=\sum_{i=1}^{N}\left(T_{i}+V_{i}\right)+\sum_{i<j}^{N} V_{i j},
$$


where $T_{i}$ and $V_{i}$ are the i-th particle's kinetic energy and potential energy, and $V_{i j}$ is the interaction energy of $i$-th and $j$-th particle, the internal energy infinitesimal change is

$$
d U=d\left(\sum_{i=1}^{N}\left(T_{i}+V_{i}\right)+\sum_{i<j}^{N} V_{i j}\right)
$$

since

$$
d Q=\sum_{i=1}^{N} d Q_{i}=\sum_{i=1}^{N} T d S_{i}=d\left(\sum_{i=1}^{N} T S_{i}\right),
$$

where $d Q_{i}$ is the $\mathrm{i}$-th particle absorbed heat, $d S_{i}$ is the $\mathrm{i}$-th entropy change. $T$ is the system temperature.

By substituting (12), (13) into (10), we have

$$
d\left(\sum_{i=1}^{N}\left(T_{i}+V_{i}\right)+\sum_{i<j}^{N} V_{i j}-T \sum_{i=1}^{N} S_{i}\right)=0 .
$$

i.e.,

$$
\sum_{i=1}^{N}\left(T_{i}+V_{i}\right)+\sum_{i<j}^{N} V_{i j}-T S=E,
$$

where $S=\sum_{i=1}^{N} S_{i}$ is system total entropy, $E$ is a constant, which can be defined as the total energy of system.

For the single particle, the (14) becomes

$$
\frac{p^{2}}{2 m}+V-T S=E
$$

where $\frac{p^{2}}{2 m}, V$ and $E$ are the single particle kinetic energy, potential energy and total energy, $S$ is the single particle microscopic entropy and $T$ is the single particle's surrounding temperature. For a classical particle, its entropy is zero, and its total energy $E=\frac{p^{2}}{2 m}+V$, i.e., its total energy is mechanical energy. For a microscopic particle, due to the wave nature, it has microscopic entropy. The microscopic particle total energy $E$ is the sum of its mechanical energy $\frac{p^{2}}{2 m}+V$ and $-T S$. We define $-T S$ as heat potential energy.

For a relativistic free particle, the total squared energy of the relativistic particle is

$$
E^{2}=m_{0}^{2} c^{4}+\vec{p}^{2} c^{2} .
$$

For a relativistic unfree particle, including interaction potential $V(r)$, its relativistic total energy and total squared energy are

$$
E=m c^{2}+V(r),
$$


and

$$
(E-V(r))^{2}=m_{0}^{2} c^{4}+\vec{p}^{2} c^{2}
$$

when a particle is in temperature $T$, its non-relativistic total energy is given by Eq. (15). Similarly, when a relativistic particle is in temperature $T$, its relativistic total energy is

$$
E=m c^{2}+V(r)-T S
$$

and its total squared energy is

$$
(E-V(r)+T S)^{2}=m_{0}^{2} c^{4}+\vec{p}^{2} c^{2}
$$

\section{Finite temperature Dirac Equation}

For the free particle of spin-1/2, we can obtain Dirac equation from Eq. (16)

$$
i \hbar \frac{\partial}{\partial t} \psi(\vec{r}, t)=\left(-i \hbar c \vec{\alpha} \cdot \nabla+\beta m_{0} c^{2}\right) \psi(\vec{r}, t)
$$

where the $\alpha_{i}(\mathrm{i}=1,2,3)$ and $\beta$ matrix are

$$
\alpha_{i}=\left(\begin{array}{cc}
0 & \sigma_{i} \\
\sigma_{i} & 0
\end{array}\right), \quad \beta=\left(\begin{array}{cc}
I & 0 \\
0 & I
\end{array}\right),
$$

and $\sigma_{i}$ are the usual $2 \times 2$ pauli matrix and $I$ the $2 \times 2$ unit matrix, the wave function $\psi$ is

$$
\psi=\left(\begin{array}{c}
\psi_{1} \\
\psi_{2} \\
\psi_{3} \\
\psi_{4}
\end{array}\right)
$$

Multiplied from the right by $\beta / c$, and with the definitions

$$
\gamma^{0}=\beta, \quad \gamma^{i}=\beta \alpha_{i} \quad(i=1,2,3)
$$

The Eq. (21) becomes

$$
i \hbar\left(\gamma^{0} \frac{\partial}{\partial x^{0}}+\gamma^{1} \frac{\partial}{\partial x^{1}}+\gamma^{2} \frac{\partial}{\partial x^{2}}+\gamma^{3} \frac{\partial}{\partial x^{3}}\right) \psi-m_{0} c \psi=0
$$

where

$$
\gamma^{i}=\left(\begin{array}{cc}
0 & \sigma_{i} \\
-\sigma_{i} & 0
\end{array}\right), \quad \gamma^{0}=\left(\begin{array}{cc}
I & 0 \\
0 & -I
\end{array}\right)
$$


The Eq. (26) can be written as

$$
\left(i \hbar \gamma^{\mu} \partial_{\mu}-m_{0} c\right) \psi=0
$$

we set $\hbar=c=1$, the Eq. (28) is

$$
\left(i \gamma^{\mu} \partial_{\mu}-m_{0}\right) \psi=0
$$

For the unfree particle of spin-1/2, we can obtain the new Dirac equation from Eq. (10)

$$
i \hbar \frac{\partial}{\partial t} \psi=\left(-i \hbar c \vec{\alpha} \cdot \nabla+\beta m_{0} c^{2}+V(r)-T S\right) \psi
$$

substituting Eq. (7) into (29), we have

$$
i \hbar \frac{\partial}{\partial t} \psi=\left(-i \hbar c \vec{\alpha} \cdot \nabla+\beta m_{0} c^{2}+V(r)+k_{w} T \ln (\bar{\psi} \psi)\right) \psi .
$$

The Eq. (30) can be simplied as

$$
\left(i \gamma^{\mu} \partial_{\mu}-m_{0}-\beta V-\beta k_{w} T \ln \bar{\psi} \psi\right) \psi=0
$$

when $V=0$, the Eq. (31) becomes

$$
\left(i \gamma^{\mu} \partial_{\mu}-m_{0}-\beta k_{w} T \ln (\bar{\psi} \psi)\right) \psi=0
$$

The Eq. (32) is the Dirac equations at finite temperature. When $T=0$, it becomes Dirac equation, i.e., the Dirac equation is the zero temperature relativistic wave equation of spin-1/2 particle.

\section{Conclusion}

In this paper, we define microscopic entropy of a single particle, and give the finite temperature Dirac equation. With the equation, we can study the quantum systems in an arbitrary temperature, such as it can be studied superconductivity mechanism, finite temperature quantum field theory and so on. 


\section{References}

[1] T. Matsubara, Prog. Theor. Phys. 14 (1955) 351.

[2] J. Schwinger, J. Math. Phys. 2 (1961) 407; J. Schwinger, Lecture Notes Of Brandeis University Summer Institute (1960).

[3] H. Umezawa, H.Matsumoto and M. Tachiki, Thero Field Dynamics and Condensed States, North-Holland, Amsterdam,1982.

[4] J. Zinn-Justin, 1989, Quantum Field Theory and Critical Phenomena, in particular chap. 36 of third ed., Clarendon Press (Oxford 1989, third ed. 1996).

[5] D. A. Kirzhnits and A. D. Linde, Phys. Lett. 42B (1979) 471; L. Dolan and R. Jackiw, Phys. Rev. D9 (1974) 3320; S. Weinberg, Phys. Rev. D9 (1974) 3357.

[6] D. J. Gross, R. D. Pisarski and L. G. Yaffe, Rev. Mod. Phys. 53 (1981) 43.

[7] N.P.Landsman and Ch.G.vanWeert, Phys.Rep. 145 (1987) 141. 